\title{
Investigation of RF transmission properties of human tissues
}

\author{
D. Werber ${ }^{1,2}$, A. Schwentner ${ }^{1}$, and E. M. Biebl ${ }^{1}$ \\ ${ }^{1}$ Technische Universität München, Fachgebiet Höchstfrequenztechnik, Arcisstraße 21, 80333 München, Germany \\ ${ }^{2}$ Technische Universität München, Lehrstuhl für Technische Elektrophysik, Germany
}

\begin{abstract}
RF transmission properties of human tissues were investigated in the frequency range from $50 \mathrm{MHz}$ to $1 \mathrm{GHz}$. This work was motivated by the increasing interest in communication links between medically active implants and external interrogator units. We investigated theoretically and experimentally the transmission loss between an implant and an external interrogator unit. We assumed that due to the size of the implant a maximum area of only $1 \mathrm{~cm}^{2}$ is available for the printed circuit antenna. The size of the external interrogator antenna is less restricted. The maximum depth of the implant beneath the surface of the body was assumed to be $10 \mathrm{~cm}$. For the simulations we took the dielectric properties of skin, fat and muscle as published in the literature. For the measurements, an artificial muscle dielectric proposed in the literature was used consisting mainly of a mixture of water, sugar and salt. In simulation and measurements the reactive part of the impedance of the antennas was compensated numerically. In simulations and measurements we obtained a transmission loss between $30 \mathrm{~dB}$ around $100 \mathrm{MHz}$ and $65 \mathrm{~dB}$ around $900 \mathrm{MHz}$.
\end{abstract}

\section{Introduction}

Active medical implants allow for a very effective and precise medical treatment. Probably the most popular active medical devices are cardiac pacemakers. But recently a new class of implants has been developed that provides local application of highly concentrated and effective drugs as beneficiary for cancer therapy, for instance. It has been demonstrated that the micro-environment of solid tumors differs from the corresponding one of normal tissues. The most ocular alteration is a shift of the microenvironmental $\mathrm{pH}$ towards acidic values. Based on these findings a implantable feedback bioactuator has been developed (Wolf et al., 1997)

Correspondence to: E. M. Biebl (biebl@tum.de)

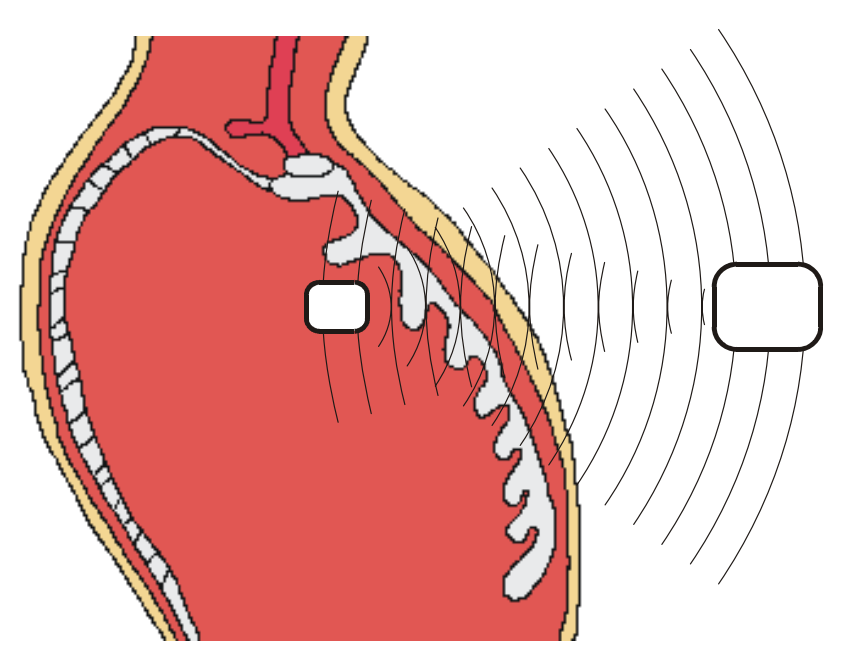

Fig. 1. Communication link between an active medical implant and an interrogator realized by RF-transmission.

that reduces the serious side effects of common chemotherapeutical treatment. A bioactuator, which is able to in vivo monitor the $\mathrm{pH}$ of the tumor microenvironment, will be used for the treatment of inoperable tumors and localized metastases, thus the dose of applied drugs can be minimized. The microsensor-based feedback bioactuator, which can be fixed directly to the tumor, consists of three main parts: the drug reservoir, the control logic and the energy supply. All components are housed in a small silicon-based device, which measures less than $10 \mathrm{~mm}$ each side. The control logic measures the $\mathrm{pH}$ and dispenses the $\mathrm{pH}$-normalizing drug directly to the tumor microenvironment impeding further malignant growth. As an additional feature to observe the implant parameters, like power and drug supply or applied drug dose, communication from outside the body to the implant shall be realized by RF transmission (Fig. 1). Not only for this special cancer therapy but for all implants a feedback mech-

Published by Copernicus GmbH on behalf of the URSI Landesausschuss in der Bundesrepublik Deutschland e.V. 

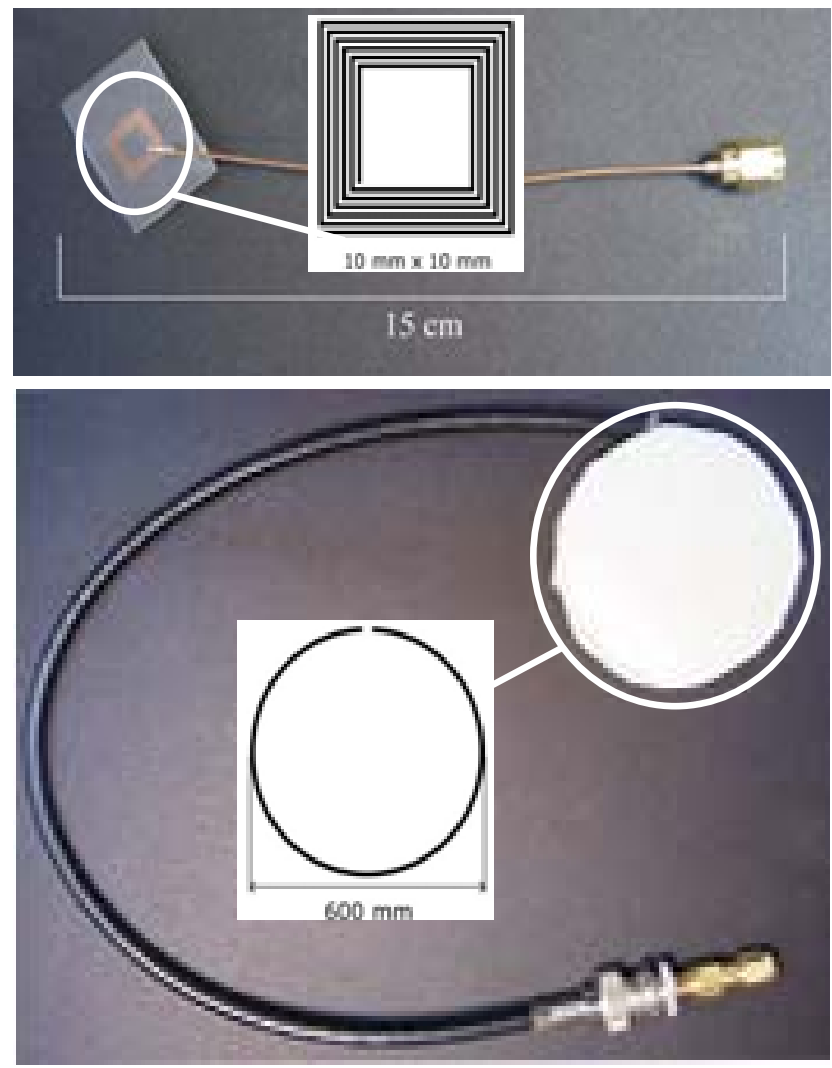

Fig. 2. (a) The implant's antenna: A quadratic helix with tenfold winding and $10 \mathrm{~mm}$ side length, printed on RT-Duroid. (b) The interrogator's antenna: A single circular loop antenna (Ø60 mm), mounted rigid foam plastic.

anism to watch and control the implant's operational status and parameters is helpful during the treatment.

Thus, the motivation of the work presented in this paper was to find design rules for communication links between small implants relatively far beneath the skin and an interrogator unit above the skin. In other words, we investigated the parameters of a RF transmission link to a small antenna surrounded and covered by relatively thick layers of highly lossy mater in order to answer the following questions: What kind of antennas is suited? What are reasonable frequencies of operation? Are there frequency restrictions (resonances)? How large is the transmission loss, i.e. what is the minimum TX power?

\section{General framework}

The implants are placed in the body consisting of many different tissues, mainly of muscles or fat. The in vivo dielectric properties of all tissues have been measured in the frequency range $10 \mathrm{~Hz}$ to $20 \mathrm{GHz}$ (Gabriel et al., 1996; http://niremf.ifac.cnr.it/tissprop). They depend strongly on the water content of the particular tissue. For frequencies
Table 1. Dielectric properties (relative permittivity and conductivity) of muscle and fat tissue at $100 \mathrm{~Hz}, 100 \mathrm{kHz}, 400 \mathrm{MHz}$ and $1 \mathrm{GHz}$.

\begin{tabular}{lcccc}
\hline & \multicolumn{2}{c}{$100 \mathrm{~Hz}$} & \multicolumn{2}{c}{$100 \mathrm{kHz}$} \\
\hline Muscle & $2.27 \mathrm{e}-1$ & $9.33 \mathrm{e} 6$ & $3.62 \mathrm{e}-1$ & 8090 \\
Fat & $2.08 \mathrm{e}-2$ & $4.57 \mathrm{e} 5$ & $2.44 \mathrm{e}-2$ & 92.9 \\
\hline \multicolumn{4}{c}{$400 \mathrm{MHz}$} & \multicolumn{2}{c}{$10 \mathrm{GHz}$} \\
Muscle & $7.96 \mathrm{e}-1$ & 57.1 & 10.6 & 42.8 \\
Fat & $4.11 \mathrm{e}-2$ & 5.58 & $5.85 \mathrm{e}-1$ & 4.6 \\
\hline
\end{tabular}

smaller than $100 \mathrm{~Hz}$, the relative permittivity can reach up to 106 of 107. For higher frequencies the relative permittivity declines gradually. The conductivity rises slowly with higher frequencies. Typical in vivo values of the relative permittivity and conductivity at selected frequencies for muscle (high water content tissue) and fat (low water content tissue) can be found in Table 1 .

Concerning the frequency regulation the implantable feedback bioactuator counts among the usage category short range device and subcategory medical implants. This category is reserved for medical implants with simplex or duplex transmission and an RF-radiated power of $25 \mu \mathrm{W}$ e.r.p.. The European frequency range proposed by ETSI lies within 402-405 MHz (ETSI EN 301 839-2). In Germany the frequency range from 403 to $405 \mathrm{MHz}$ is reserved for medical applications.

\section{Antennas}

The size of the implant's antenna is restricted by the dimensions of the implant and may not exceed an area of $1 \mathrm{~cm} \times 1 \mathrm{~cm}$. The size of the interrogator's antenna is only determined by ease of handling. From the gradient of permittivity and conductivity of human tissue it is clear that a frequency of operation beyond about $1 \mathrm{GHz}$ is not reasonable. Thus, the distance between the two antennas and the area for the inner body antenna are electrically small. Because of their good inductive coupling in the near field and small susceptibility to dielectric losses, loop antennas were used for both antennas. The implant's antenna is a quadratic helix with tenfold winding and $10 \mathrm{~mm}$ side length, printed on Roger's RT-Duroid and feed via a semi-rigid coaxial cable. In Fig. 2a the layout and a picture of the implant's antenna is given. The interrogator's antenna is a single circular loop antenna $(\emptyset 60 \mathrm{~mm})$ wound around a on rigid foam plastic. A sketch and a photo of the interrogator's antenna is shown in Fig. 2b. 


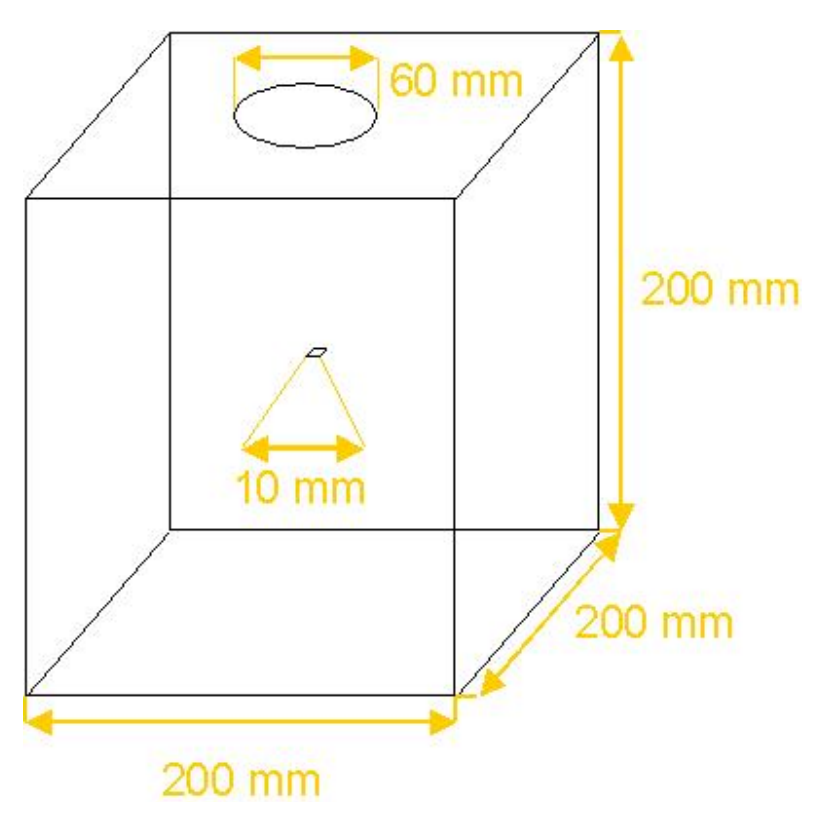

Fig. 3. Model used for simulation: A spiral antenna in the centre of a cubic muscle tissue and a loop antenna at the surface of the cube.

\section{Simulation}

From the dielectric properties of human tissues we concluded that a suitable carrier frequency for communication with an implant would be between $50 \mathrm{MHz}$ and $1 \mathrm{GHz}$, corresponding to free-air wavelength from $6 \mathrm{~m}$ to $30 \mathrm{~cm}$. Since the relevant dimensions of a human body are in the same order of magnitude, we expected resonances and limited repeatability of measurements due to in homogeneities and irregularity of the human body. Even for measurements with a phantom muscle the influence of the surrounding environment was hard to control. Thus, we simulated a transmission link between a spiral antenna in the centre of a cubic muscle tissue and an interrogator loop antenna at the surface of the cube (see Fig. 3). The edge length of the cube was $20 \mathrm{~cm}$ and the cube was surrounded by vacuum. The simulation was made by use of Agilent's HFSS and the whole set of S-parameters were calculated for each frequency point. Since the significant reactive part of the antennas' impedance would be compensated by the TX/RX circuits in a technical solution, we normalized the transmission loss to an impedance of $50 \Omega$ assuming lossless matching circuits.

Figure 4 shows the calculated transmission loss. The loss increases almost monotonically from about $43 \mathrm{~dB}$ at $50 \mathrm{MHz}$ to $63 \mathrm{~dB}$ at $700 \mathrm{MHz}$. Two minor resonances are observed that correspond fairly to the size of the cube and the permittivity. In order to check how skin and fat-layer influence the result a similar setup was analysed: In a cube of the same size at all six surfaces a $5 \mathrm{~mm}$ thick layer of skin and beneath a $3 \mathrm{~cm}$ thick layer of fat was assumed. Inside the remaining cubic space with an edge length of $13 \mathrm{~cm}$ was filled with muscle

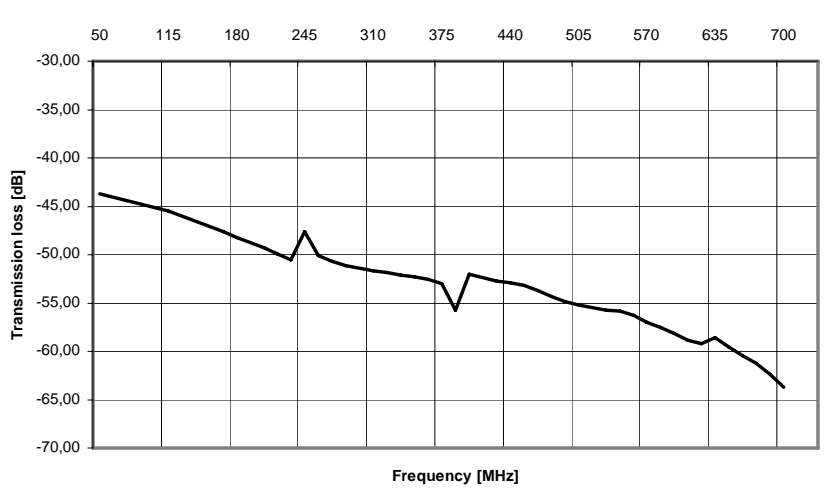

Fig. 4. Calculated transmission loss.

tissue. Since the simulation of this structure required much more CPU time, we calculated the S-parameters only for a few frequencies for reference. The values of the transmission loss were a little smaller compared to the simple model containing only muscle tissue, since muscle tissue exhibits the largest loss tangent.

\section{Measurements}

To simplify the measurement the complex tissue mix of the body has been reduced to muscle tissue only. Instead of human muscle tissue at body temperature phantom muscle tissue at room temperature is utilized. This phantom muscle tissue exhibits comparable dielectric properties to human muscle tissue in the range from $100 \mathrm{MHz}$ to $1 \mathrm{GHz}$ (Hartsgrove et al., 1987). It consists of water $(52,4 \%), \mathrm{NaCl}(1,4 \%)$, sugar $(45,0 \%)$, HEC $(1,0 \%)$ and bactericide $(0,1 \%)$. This mixture was filled in plastic freezer bags and hermetically sealed. Each of these phantom cushions was about $30 \mathrm{~cm} \times 30 \mathrm{~cm}$ in size and $25 \mathrm{~mm}$ thick.

For the measurements a network analyzer Agilent 8753C and a S-parameter test set Agilent 85047 A was used. Calibration of this VNA was done at the coaxial connectors by means of a 2-port full calibration procedure. Then, the complete set of S-parameters were measured from $100 \mathrm{MHz}$ to $1 \mathrm{GHz}$. From these S-parameter sets the matched transmission loss was calculated, i.e. lossless matching circuits were assumed.

In the first measurement the transmission through phantom muscle tissue was measured. One, two or three filled cushions, i.e. 25,50 or $75 \mathrm{~mm}$ phantom muscle tissue, respectively, were put in between the antennas (Fig. 5). Figure 6 shows the transmission loss through phantom muscle tissue. In the range from $100 \mathrm{MHz}$ to $1 \mathrm{GHz}$ the attenuation increases from $20 \mathrm{~dB}$ to $60 \mathrm{~dB}$. Though a couple of minor resonances can be observed transmission is feasible in the whole frequency range. In general, the transmission losses are somewhat smaller than the calculated values. As expected the transmission loss increases with increasing thick- 


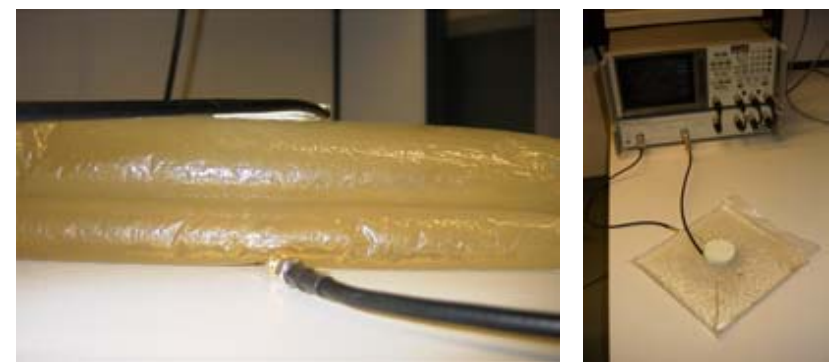

Fig. 5. Experimental setup: Phantom muscle tissue cushions between of the implant's and the interrogator's antenna.

attenuation of phantom muscle tissue

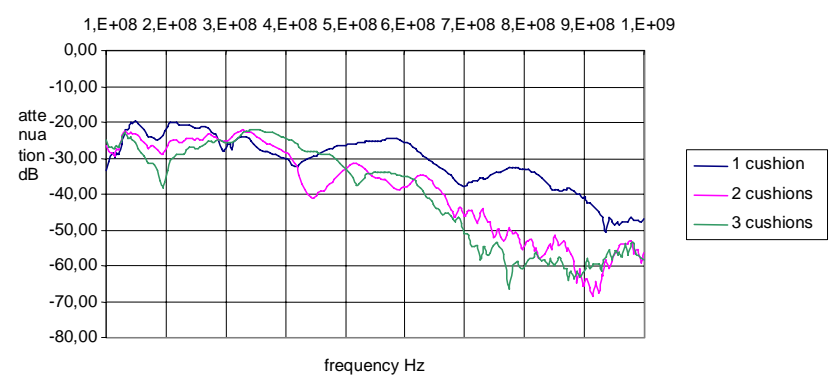

Fig. 6. Measured transmission loss characteristic for 25,50 , and $75 \mathrm{~mm}$ thick phantom muscle tissue

ness of the phantom muscle tissue by about $5 \mathrm{db} @ 100 \mathrm{MHz}$ to about $10 \mathrm{~dB} @ 1 \mathrm{GHz}$ per $25 \mathrm{~mm}$.

To compare the transmission characteristics of phantom muscle tissue with the transmission of a human tissue sequence the transmission through a human abdomen was investigated. The two antennas were placed on the skin at opposite sides of a human abdomen, Two measurements have been carried out: One with about $150 \mathrm{~mm}$ distance of the two antennas, and the other with about $200 \mathrm{~mm}$ distance (Fig. 7). In general, the loss is significantly smaller than expected from the measurements with the phantom muscle tissue. The reason is not quite clear. Probably, fields travelling around the body may cause this. The transmission loss for a distance of $150 \mathrm{~mm}$ is roughly between 20 and $50 \mathrm{~dB}$, and for $200 \mathrm{~mm}$ distance between 30 and $70 \mathrm{~dB}$.

\section{Conclusions}

The transmission of RF (100 MHz to $1 \mathrm{GHz})$ through human tissue was investigated. Simulations and measurements showed that a total transmission loss between 20 and $60 \mathrm{~dB}$ has to be expected for a distance of $10 \mathrm{~cm}$. The transmission loss increases with frequency, moreover small resonances were observed, but obviously transmission is possible within this whole frequency range. Usage of the frequency bands reserved by the regulatory authorities around $400 \mathrm{MHz}$ seems

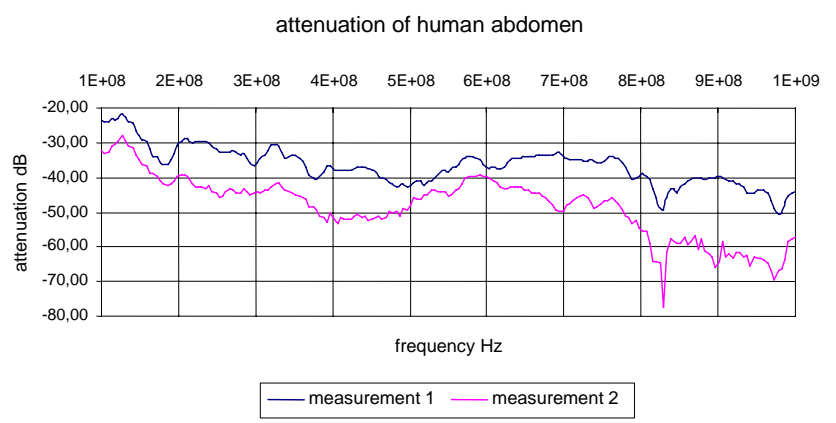

Fig. 7. Measured transmission loss for (roughly) 150 and $200 \mathrm{~mm}$ thick human abdomen.

reasonable. But regarding the transmission properties these bands exhibit no particular advantage nor disadvantage.

Typical transceiver in the $433 \mathrm{MHz}$ ISM band for telemetry exhibit a sensitivity of about $-100 \mathrm{dBm}$ (4800 baud, $\mathrm{SNR}=20 \mathrm{~dB}$ ). Thus, a output power of the implant's transmitter of $1 \mu \mathrm{W}$ is sufficient to establish reliable communication resulting in a very small additional load to the energy budget of the implant. This small value of radiated power is well below the limits set by the regulatory authorities and, moreover, negative side effects of the radiation can be ruled out.

In an overall evaluation, our investigations demonstrate that communication links to small active implants are feasible.

\section{References}

Gabriel, C., Gabriel, S.: Dielectric properties of biological tissues: I-III, Physics in Medicine and Biology, 41, 2231-22903, 1996.

Hartsgrove, G., Kraszewski, A., and Surowiec, A.: Simulated Biological Materials for Electromagnetic Radiation Absorption Studies, Bioelectromagnetics, 8, 29-36, 1987.

Wolf, B., Kraus, M., and Sieben, U.: Potential of microsensor-based feedback bioactuators for biophysical cancer treatment, Biosensors and Bioelectronics, 12, 301-309, 1997. 